\title{
Overcoming Brazil's monumental COVID-19 failure: an urgent call to action
}

To the Editor-2.7\% of the world's population lives in Brazil, a country widely known for its world-class soccer, its touristic Carnival and its exuberant-yet under threat-Amazon forest. Unfortunately, Brazil is now also known as the global epicenter for COVID-19. As of 10 April 2021, $12.0 \%$ of all global COVID-19 deaths took place in Brazil ${ }^{1}$, and throughout March 2021, this figure was $23.5 \%$.

On the one hand, record-breaking death tolls are announced daily, and bed occupancy in intensive care units (ICUs) exceeds $100 \%$ in many states due to waiting lists, as supplies of oxygen and medicines are being depleted. On the other hand, the country's president walks in open and closed spaces without a face mask, takes selfies with equally unprotected fans, ignores basic scientific recommendations, and continues to represent the greatest threat to Brazil's response to COVID- $19^{2}$.

With the causative coronavirus circulating freely, it is no coincidence that a new variant ${ }^{3}$ has been identified in Japan in a traveler who arrived from Brazil, and it rapidly became the dominant strain throughout the country. In addition to being more transmissible and causing more-severe illness, the P.1 variant is also affecting young people to a much greater extent than the original strain did. The P.1 variant caused a new epidemic wave in the Amazon region, which had also been the first part of Brazil affected by the first wave in early $2020\left(\right.$ ref. $\left.^{4}\right)$. As the variant spread in the city of Manaus, hospital beds became fully occupied and countless patients died due to the limited availability of hospital oxygen supplies, the federal government's response was to send medical teams to the city to promote ineffective early treatment (with hydroxychloroquine, ivermectin and other drugs), and then to contribute to the spread of the new variant by referring critically ill patients to most Brazilian state capitals. No consideration was given to the establishment of a cordon sanitaire or to bring in medical teams and mobile hospitals to help contain the spread of the new variant.

Denial of science has been a constant in the response of the federal government to the pandemic. The country's largest epidemiological study on COVID $-19^{4}$ was discontinued by the Ministry of Healthwhich is headed by an Army general-in July 2020, after the study identified marked regional, ethnic and socioeconomic disparities in antibody prevalence throughout the country ${ }^{5}$.

Without ignoring the past, it is now time to look ahead. First, Brazil needs to leave behind scientific 'negationism. The country's response to the pandemic needs to be driven by science ${ }^{6}$. Second, Brazil needs a paradigm change. The current clinical approach prioritizes increasing the number of hospital beds-in particular, ICU beds-indefinitely, as well as the widespread promotion of useless drugs for early treatment. Such an approach needs to be replaced by population-level measures to stop the spread of the virus, such as the widespread use of masks, evidence-based social-distancing strategies, large-scale testing and contact-tracing policies. Several state and municipal governments have adopted such measures, even in defiance of federal recommendations, but in most places, lockdown policies have been short-lived, the use of face masks is not mandatory, and general compliance by the population is patchy-which is not surprising, given the poor example provided by the country's president.

After several months of denying the importance of vaccines and failing to procure doses, the federal government has finally realized the importance of vaccination as a key strategy for controlling the pandemic. The most widely available vaccine in the country is CoronaVac, which has an efficacy level below that of alternative vaccines, such as the vaccine from Pfizer, which the government rejected in mid-2020 despite being offered this vaccine. Even the current supplies of CoronaVac and (to a lesser extent) the vaccine from OxfordAstraZeneca are thus far clearly insufficient.

There are two targets to be achieved over the coming weeks. First, Brazil needs to average 1.5 million doses of vaccines administered per day to reach full coverage of people 18 years of age and older by the end of the year. As of 10 April 2021, on average, 278,000 daily vaccine doses had been delivered in the first 93 days of vaccinationthat is, just over one dose per thousand Brazilians. The pace of immunization has accelerated more recently but is still far from the above-mentioned target.

Second, there is an urgent need for a strictly enforced 3-week national lockdown, a strategy that will save thousands of lives and will within a few months help rebuild the country's devastated economy. Given the importance of the informal economy, civil society and government sectors, including the army, must be involved in the distribution of food rations to the poorest families during the lockdown. Realistically, enforcement of such a lockdown will face many barriers, from the opposition of the national government to pressure by local businesses.

The P.1 variant is a clear indication that Brazil is uniquely posed to become a global 'variant factory' and thus represents a threat to the whole world. International organizations and vaccine producers should prioritize Brazil in vaccine-distribution efforts. Help is also needed with drugsparticularly for use in ICUs-and personal protective equipment.

Political pressure from outside Brazil is also important. Just like such pressure was applied to environmental conservation in Brazil, the current threat to global public health should be recognized and acted upon by foreign trade partners. Brazilian businesspeople were strong supporters of President Bolsonaro's election and, although such support has waned over time, they still represent a strong voice in guiding national decisions-surely, and unfortunately, much stronger than the voice of the scientific community.

Pedro C. Hallal (D) and Cesar G. Victora (D) Postgraduate Program in Epidemiology, Federal University of Pelotas, Pelotas, Brazil.

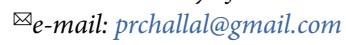

Published online: 6 May 2021

https://doi.org/10.1038/s41591-021-01353-2

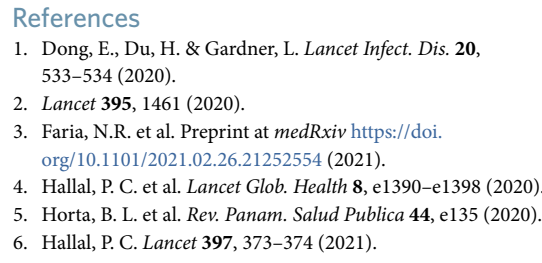
the final version on the basis of feedback from C.G.V.; and both authors have seen and approved the submitted version.

Competing interests

The authors declare no competing interests. 